\title{
Computational Aerothermodynamic Simulation Issues on Unstructured Grids
}

\author{
Peter A. Gnoffo ${ }^{*}$ and Jeffery A. White ${ }^{\dagger}$ \\ NASA Langley Research Center, Hampton, VA 23681
}

\begin{abstract}
The synthesis of physical models for gas chemistry and turbulence from the structured grid codes LAURA and VULCAN into the unstructured grid code FUN3D is described. A directionally Symmetric, Total Variation Diminishing (STVD) algorithm and an entropy fix (eigenvalue limiter) keyed to local cell Reynolds number are introduced to improve solution quality for hypersonic aeroheating applications. A simple grid-adaptation procedure is incorporated within the flow solver. Simulations of flow over an ellipsoid (perfect gas, inviscid), Shuttle Orbiter (viscous, chemical nonequilibrium) and comparisons to the structured grid solvers LAURA (cylinder, Shuttle Orbiter) and VULCAN (flat plate) are presented to show current capabilities. The quality of heating in 3D stagnation regions is very sensitive to algorithm options - in general, high aspect ratio tetrahedral elements complicate the simulation of high Reynolds number, viscous flow as compared to locally structured meshes aligned with the flow.
\end{abstract}

\section{Nomenclature}

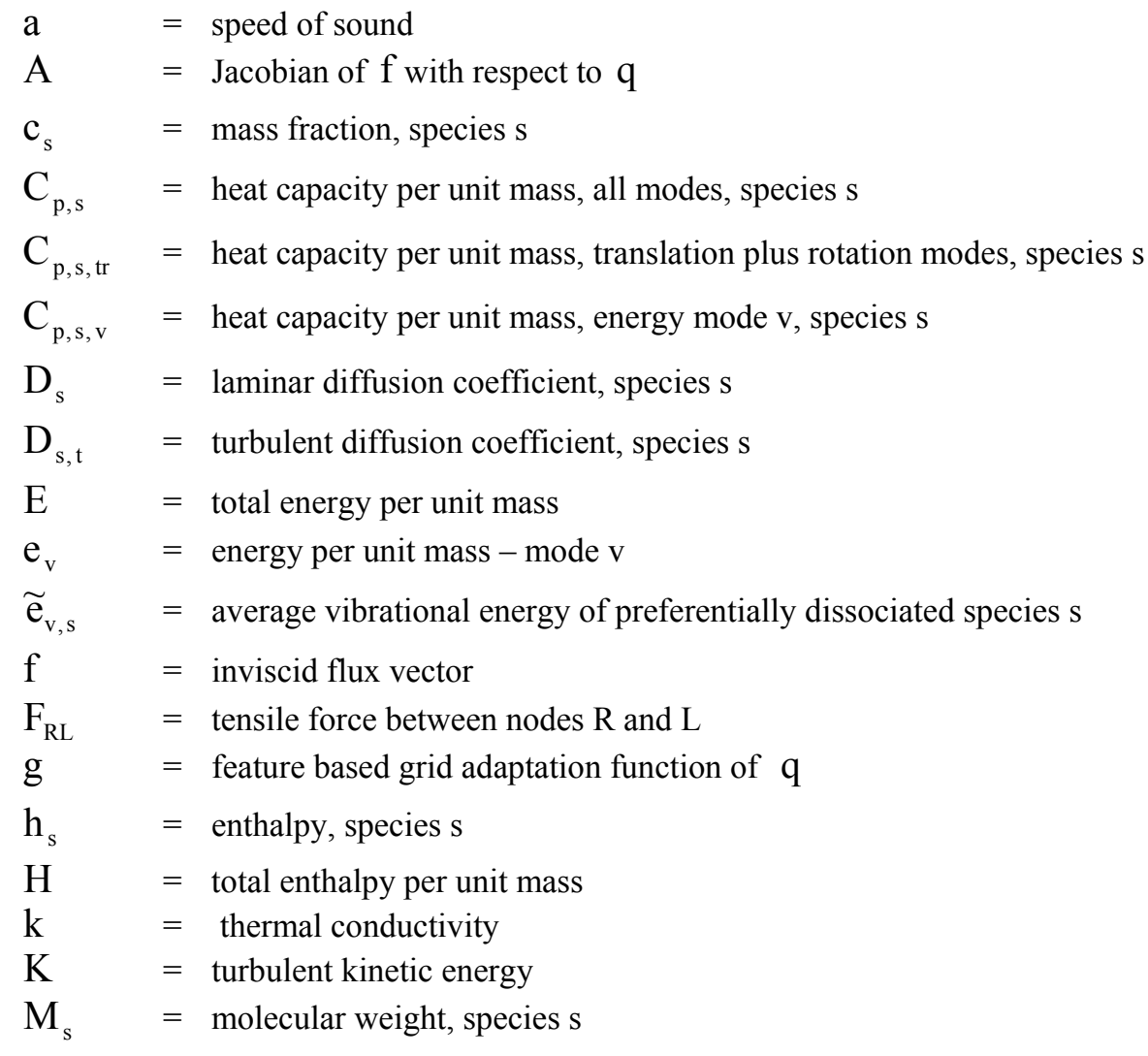

\footnotetext{
* Senior Research Engineer, Aerothermodynamics Branch, MS 408A, Fellow.

${ }^{\dagger}$ Senior Research Engineer, Hypersonic Airbreathing Propulsion Branch, MS 168, Senior Member.
} 


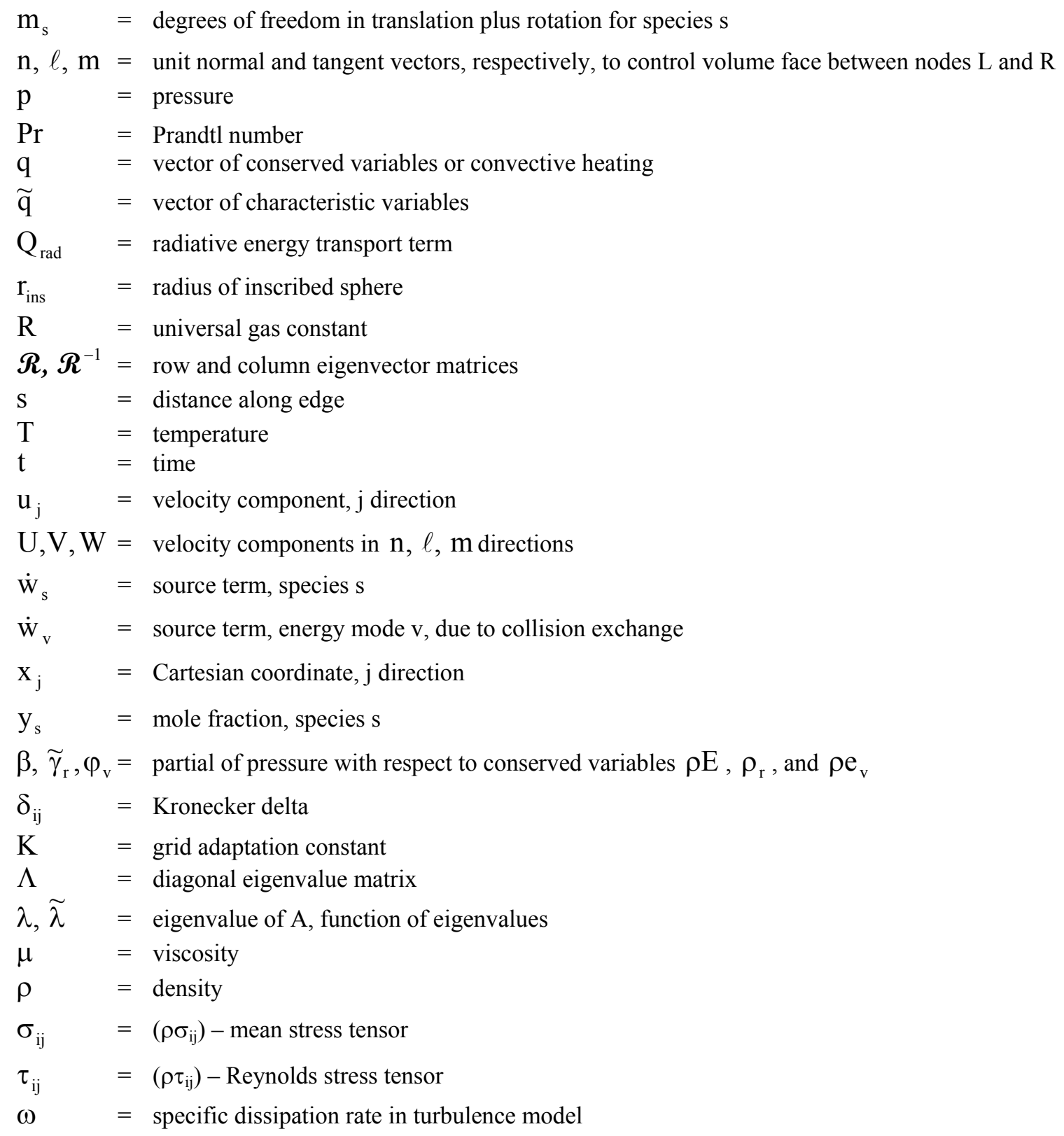

\section{Subscripts}

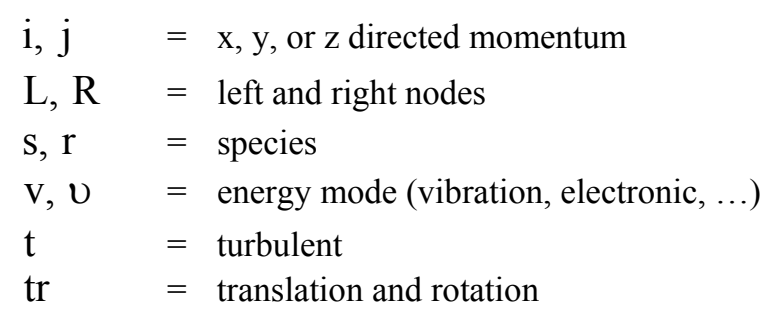




\section{Introduction}

$\mathrm{C}$

Computational fluid dynamics (CFD) is the numerical simulation of flowfields through the approximate solution of the governing partial differential equations for mass, momentum, and energy conservation coupled with the appropriate relations for thermodynamic and transport properties. Aerothermodynamics is the branch of fluid dynamics that focuses on the effects of thermodynamic and transport models on aerodynamics and heat transfer. It is especially focused on conditions of hypersonic velocities where the energy content and exchange between kinetic, internal, and chemical modes in the flow precludes the otherwise common use of calorically perfect gas assumptions. Computational aerothermodynamics is therefore defined in exactly the same manner as CFD, with the added emphasis that high temperature gas effects on pressure, skin friction, and heat transfer are included in the numerical simulation. The fundamental role of computational aerothermodynamics is the simulation of aerodynamic forces and heating for external and internal high speed flows. Reference 1 presents a review of recent applications for access to space and planetary missions.

The approximate solution of the governing equations requires that the domain be subdivided into many small control volumes. The accuracy of the solution depends upon a variety of factors; the most critical factors are the size of each control volume, the orientation of its boundaries relative to a variety of flow features, and the order of accuracy of the discretization. At the risk of belaboring the obvious, note that hypersonic flows routinely involve extremes of pressure, density, and temperature separated by shocks, expansions, shear layers, and boundary layers. These extremes in conditions and topology of flow structure complicate computational aerothermodynamic simulation. The ability to orient a grid with evolving flow structures is particularly challenging. For example, accurate simulation of heat transfer requires adequate resolution of the boundary layer (or merged layer) and accurate representation of conditions at the boundary-layer edge. Conditions at the boundary-layer edge in turn, particularly in the stagnation region of hypersonic flows, are dependent on entropy carried along streamlines from the shock. Any irregularities in the captured shock create associated irregularities in entropy that feed the rest of the domain. Further complicating the simulation is the fact that the natural stability properties of upwind schemes so often used in hypersonic applications are in turn most poorly realized in the broad, stagnation region of blunt bodies where system eigenvalues are small.

Nevertheless, computational aerothermodynamic tools that generally do a good job in handling the difficulties of steady, laminar, hypersonic, blunt body and attached flow simulation have evolved over the past 15 years. The greatest uncertainty in such applications is the validation of physical models for a host of non-equilibrium processes $^{2}$. Greater challenges are encountered in the simulation of separated flows, complex shock-shock and shock - boundary-layer interactions, prediction of transition, time-dependent flows, and plasma flows. The application of unstructured grid methods for such three-dimensional simulations (even the relatively simple blunt body problem) has proven to be especially challenging when heat transfer is required as will be discussed herein. Algorithms observed to improve simulation quality are presented, though significant problems remain.

The synthesis of the physical models within the structured codes LAURA ${ }^{3}$ (focus on external, hypersonic flow simulations) and VULCAN ${ }^{4}$ (focus on internal, scramjet flow simulations) into the unstructured flow solver FUN3D ${ }^{5}$ (perfect gas flow simulations with adjoint-based design and optimization capabilities) has proceeded within the High Energy Flow Solver Synthesis ${ }^{6}$ (HEFSS) project. The ultimate goal of this project is to develop robust schemes with quantified uncertainties requiring minimal user intervention for aerothermodynamic applications. This capability exists as a generic gas model option utilizing HEFSS modules within the code FUN3D.

\section{Grid}

The HEFSS team is investing in unstructured grid technology, including the use of mixed elements - tetrahedron (tets), prisms, pyramids, and hexahedron (hexes) - as the underlying support for future hypersonic flow simulation. We defer any investment in patched or overset multiblock structured grid systems at this time. The rationale for this approach is based on our assessment ${ }^{7}$ of current and anticipated future capabilities for grid generation and grid adaptation. This assessment considers: (1) efficiency in the initial generation of a "reasonable" grid over complex configurations; (2) ability to adapt the grid to flow structures (steady and time-dependent) and changes in parameterized structures on the vehicles; and (3) solution quality and efficiency on a "good" grid using best available algorithms. A "reasonable" grid is one that will accommodate a converged solution with at least approximate resolution of flow structures. It provides an initial condition for future adaptation and refinement. A "good" grid is one that will produce a grid converged solution such that an additional refinement will result in 
dependent variable changes within user specified tolerances. The three criteria defined above are discussed in more detail in a related paper $^{7}$ - especially as regards simulations of hypersonic flows.

Only grids composed entirely of tets are used in the present work. We forego the usual practice of resolving the near wall boundary layer regions with hexes or prisms. It is not clear if structured or semi-structured grids are required to accurately simulate convective heating and shear. (We have been unable to find published reports with a focus on heating or shear that have used tets all the way to the wall.) We start here with a view that a requirement for a semi-structured grid across boundary layers and free shear layers is a constraint that, at a minimum, complicates grid generation and adaptation algorithms. If we find that tets are unacceptable for boundary layer resolution we must invest in grid adaptation algorithms that will accommodate resolution of free shear layers (and possibly shock waves) with high-aspect-ratio, semi-structured cells properly aligned with the flow features. For now, we look to extract as much capability as possible using only tets.

\section{Physical Models}

The compressible Reynolds averaged equations for species continuity, momentum, total energy, and modal (vibration, electronic) energy conservation are written as:

$$
\begin{gathered}
\frac{\partial}{\partial t}\left(\rho c_{s}\right)+\frac{\partial}{\partial x_{j}}\left(\rho c_{s} u_{j}\right)=\frac{\partial}{\partial x_{j}}\left(\rho\left(D_{s}+D_{s, t}\right) \frac{\partial}{\partial x_{j}}\left(y_{s}\right)\right)+\dot{w}_{s} \\
\frac{\partial}{\partial t}\left(\rho u_{i}\right)+\frac{\partial}{\partial x_{j}}\left(\rho u_{i} u_{j}+p \delta_{i j}\right)=\frac{\partial}{\partial x_{j}}\left(\rho \sigma_{i j}\right)-\frac{\partial}{\partial x_{j}}\left(\rho \tau_{i j}\right) \\
\frac{\partial}{\partial t}(\rho E)+\frac{\partial}{\partial x_{j}}\left(\rho u_{j} H\right)=\frac{\partial}{\partial x_{j}}\left(\left(k+k_{t}\right) \frac{\partial T}{\partial x_{j}}+\left(k_{v}+k_{v t}\right) \frac{\partial T_{v}}{\partial x_{j}}+u_{i}\left(\frac{\partial}{\partial x_{j}}\left(\rho \sigma_{i j}-\rho \tau_{i j}\right)\right)\right) \\
+\frac{\partial}{\partial x_{j}}\left(\rho\left(D_{s}+D_{s, t}\right) h_{s} \frac{\partial}{\partial x_{j}}\left(y_{s}\right)\right)+\frac{\partial}{\partial x_{j}}\left(\left(\frac{\mu}{P r}+\frac{\mu_{t}}{\operatorname{Pr}_{t}}\right) \frac{\partial K}{\partial x_{j}}\right)-Q_{r a d} \\
\frac{\partial}{\partial t}\left(\rho e_{v}\right)+\frac{\partial}{\partial x_{j}}\left(\rho u_{j} e_{v}\right)=\frac{\partial}{\partial x_{j}}\left(\left(k_{v}+k_{v t}\right) \frac{\partial T_{v}}{\partial x_{j}}+\left(D_{s}+D_{s, t}\right) h_{v s} \frac{\partial}{\partial x_{j}}\left(y_{s}\right)\right) \\
+\dot{\mathrm{w}}_{v}+\dot{\mathrm{w}}_{s} \widetilde{e}_{v s}-Q_{r a d, v}
\end{gathered}
$$

where $\rho \sigma_{\mathrm{ij}}=\mu\left[\left(\frac{\partial \mathrm{u}_{\mathrm{i}}}{\partial \mathrm{x}_{\mathrm{j}}}+\frac{\partial \mathrm{u}_{\mathrm{j}}}{\partial \mathrm{x}_{\mathrm{i}}}\right)-\frac{2}{3} \frac{\partial \mathrm{u}_{\mathrm{k}}}{\partial \mathrm{x}_{\mathrm{k}}} \delta_{\mathrm{ij}}\right]$ is the mean stress tensor. Repeated indices indicate summation over direction ( $\mathrm{j}$ or $\mathrm{k})$, species $(\mathrm{s})$, or energy mode $(\mathrm{v})$. The Reynolds stress tensor, $\rho \tau_{i j}$, is a product of the averaging process and must be modeled to close the system of equations.

\section{A. Thermodynamic Models}

Three options for the equation of state: (1) calorically perfect, (2) chemical equilibrium, and (3) chemical nonequilibrium mixture of thermally perfect gases are accommodated within the HEFSS thermodynamics module. In the case of chemical equilibrium, $\mathrm{p}(\rho, \mathrm{e})$ is available from curve fits for air. (Other gas mixtures with constant elemental mass fraction may be accommodated in like manner.) In the case of chemical nonequilibrium, a mixture of thermally perfect gases with $p=\rho_{s} R T / M_{s}$, is assumed. Curve fits for heat capacity of species s, $C_{p, s}(T)$, 
and related thermodynamic properties are available in the literature. ${ }^{8,9}$ In the event that a temperature falls outside the curve fit range it is assumed that $\mathrm{C}_{\mathrm{p}, \mathrm{s}}(\mathrm{T})=\mathrm{C}_{\mathrm{p}, \mathrm{s}}\left(\mathrm{T}_{\min }\right)$ if $\mathrm{T}<\mathrm{T}_{\min }$ and $\mathrm{C}_{\mathrm{p}, \mathrm{s}}(\mathrm{T})=\mathrm{C}_{\mathrm{p}, \mathrm{s}}\left(\mathrm{T}_{\max }\right)$ if $\mathrm{T}>\mathrm{T}_{\max }$.

Thermal nonequilibrium is simulated with a two-temperature mode ${ }^{10}$ in which heavy particle translation and rotation energy modes are assumed in equilibrium at temperature $\mathrm{T}$ and vibration, electronic, and free electron translation energy modes are assumed in equilibrium at temperature $T_{v}$. The species thermodynamic curve fits are still employed in this model by assuming that translation and rotation modes are fully engaged: $\mathrm{C}_{\mathrm{p}, \mathrm{s}, \mathrm{tr}}=\frac{\left(\mathrm{m}_{\mathrm{s}}+2\right)}{2} \frac{\mathrm{R}}{\mathrm{M}_{\mathrm{s}}}$ where $\mathrm{m}_{\mathrm{s}}$ is the number of degrees of freedom in translation (3) plus rotation (2 for diatomic molecule) for species $\mathrm{s}$. Vibration-electronic heat capacity is then defined: $\mathrm{C}_{\mathrm{p}, \mathrm{s}, \mathrm{v}}=\mathrm{C}_{\mathrm{p}, \mathrm{s}}\left(\mathrm{T}_{\mathrm{v}}\right)-\mathrm{C}_{\mathrm{p}, \mathrm{s}, \mathrm{tr}}$. These relations form the basis for computation of the Jacobian of pressure and temperature with respect to conserved variables. ${ }^{11}$

B. Source Terms

Species source terms: $\quad \dot{\mathrm{w}}_{\mathrm{s}}=\mathrm{M}_{\mathrm{s}} \sum_{\mathrm{r}=1}^{\mathrm{N}_{\mathrm{r}}}\left(\beta_{\mathrm{s}, \mathrm{r}}-\alpha_{\mathrm{s}, \mathrm{r}}\right)\left(\mathrm{R}_{\mathrm{f}, \mathrm{r}}-\mathrm{R}_{\mathrm{b}, \mathrm{r}}\right)$ with $\mathrm{R}_{\mathrm{f}, \mathrm{r}}=\mathrm{k}_{\mathrm{f}, \mathrm{r}} \prod_{\mathrm{s}=1}^{\mathrm{N}_{\mathrm{s}}}\left(\frac{\rho_{\mathrm{s}}}{\mathrm{M}_{\mathrm{s}}}\right)^{\alpha_{\mathrm{s}, \mathrm{r}}}$, $\mathrm{R}_{\mathrm{b}, \mathrm{r}}=\mathrm{k}_{\mathrm{b}, \mathrm{r}} \prod_{\mathrm{s}=1}^{\mathrm{N}_{\mathrm{s}}}\left(\frac{\rho_{\mathrm{s}}}{\mathrm{M}_{\mathrm{s}}}\right)^{\beta_{\mathrm{s}, \mathrm{r}}}$ where $\alpha_{\mathrm{s}, \mathrm{r}}$ and $\beta_{\mathrm{s}, \mathrm{r}}$ are stoichiometric coefficients for species $\mathrm{s}$ as reactants and products, respectively, in reaction $\mathrm{r}$. Reaction sets are taken from References 12 and 13. The reaction rate coefficients ${ }^{12,13}$ are functions of temperature: $\mathrm{k}_{\mathrm{f}, \mathrm{r}}=\mathrm{C}_{\mathrm{r}} \mathrm{T}_{\mathrm{q}}^{\eta_{\mathrm{r}}} \exp \left(-\mathrm{E}_{\mathrm{r}} / \boldsymbol{k} \mathrm{T}_{\mathrm{q}}\right)$ and $\mathrm{k}_{\mathrm{b}, \mathrm{r}}=\mathrm{k}_{\mathrm{f}, \mathrm{r}}(\mathrm{T}) / \mathrm{K}_{\mathrm{c}, \mathrm{r}}$. The equilibrium constant, $K_{c, r}$, is derived from thermodynamic curve fit data for each species in the reaction. The effective temperature for a reaction, $T_{q}$, is equal to $T$ in the case of thermal equilibrium and exchange / shuffle reactions in thermal nonequilibrium. It is equal to $T^{0.7} T_{v}^{0.3}$ for dissociation reactions and equal to $T_{v}$ for electron impact ionization as an empirical approximation to the effect of thermal nonequilibrium in these processes. A minimum rate controlling temperature may be specified to preclude stiffness related convergence problems. These source terms and their Jacobians with respect to conserved variables are computed within the HEFSS kinetics module. The effect of turbulence models on kinetic rates ${ }^{4}$ are not yet modeled.

Energy mode source terms: $\dot{\mathrm{w}}_{\mathrm{v}}=\frac{\rho_{\mathrm{s}}\left(\mathrm{e}_{\mathrm{v}, \mathrm{s}}^{*}-\mathrm{e}_{\mathrm{v}, \mathrm{s}}\right)}{\tau_{\mathrm{s}}}-\dot{\mathrm{n}}_{\mathrm{e}, \mathrm{s}} \hat{\mathrm{I}}_{\mathrm{s}}$. The first term represents the relaxation between vibration-electronic modes and translation-rotation modes (combine terms 6 and 7 of Equation 16 in Reference 11) and $\mathrm{e}_{\mathrm{v}, \mathrm{s}}^{*}$ is the vibration-electronic energy for species $\mathrm{s}$ at thermal equilibrium conditions $\left(T_{v}=T\right)$. The second term represents the energy loss due to electron impact ionization. ${ }^{11}$ The depletion of vibrational energy through dissociation uses the term $\widetilde{\mathrm{e}}_{\mathrm{vs}}$ to model the (possibly) preferential vibration energy level of dissociating molecules. These source terms and their Jacobians with respect to conserved variables are computed within the HEFSS thermal relaxation module.

\section{Molecular Transport}

Transport properties for a perfect gas are calculated from Sutherland's law and constant Prandtl number. Curve fits are used for equilibrium air $^{14}$. Polynomial fits for collision cross sections of species pairs as a function of temperature ${ }^{15}$ are used to compute transport properties for gas in chemical nonequilibrium. ${ }^{11}$ The binary diffusion approximation is employed in Eq. 1. The summation of the diffusion term in Eq. 1 over all species should equal zero. A mass weighted correction term as defined in Reference 16 enforces this zero sum condition. 


\section{Turbulence Models}

Linear eddy viscosity models assume that the shear stress angle and the mean flow angle are the same. Rumsey and Gatski ${ }^{17}$ investigated the effect of $\mathrm{K}-\varepsilon$ and $\mathrm{K}-\omega$ forms (both accommodated within HEFSS) of twoequation models on a multi-element airfoil. They demonstrated that models based on the $\mathrm{K}-\varepsilon$ formulation were typically deficient in predicting adverse pressure gradient flows. Models based on the $\mathrm{K}-\omega$ formulation provided a better prediction of adverse pressure gradient flows. Only the $K-\omega$ linear eddy viscosity model of Wilcox ${ }^{18}$ is tested here.

The turbulent kinetic energy and specific dissipation rate are calculated by solving the following transport equations fully coupled to the species, momentum, and energy conservation:

$$
\begin{aligned}
& \frac{\partial}{\partial t}(\rho K)+\frac{\partial}{\partial x_{j}}\left(\rho u_{j} K\right)=\rho P-f_{\beta^{\prime}} \rho \omega K+\frac{\partial}{\partial x_{j}}\left[\left(\mu+\frac{\mu_{t}^{*}}{\sigma_{K}}\right) \frac{\partial K}{\partial x_{j}}\right] \\
& \frac{\partial}{\partial t}(\rho \omega)+\frac{\partial}{\partial x_{j}}\left(\rho u_{j} \omega\right)=\gamma \frac{\rho \omega}{K} P-\beta \rho \omega^{2}+\frac{\partial}{\partial x_{j}}\left[\left(\mu+\frac{\mu_{t}^{*}}{\sigma_{\omega}}\right) \frac{\partial \omega}{\partial x_{j}}\right]
\end{aligned}
$$

where production term $\mathrm{P}$ and the coefficients $\sigma_{\mathrm{K}}, \sigma_{\omega}, \gamma, \beta, \mathrm{f}_{\beta^{\prime}}$ are derivable from those in Wilcox ${ }^{18}$.

\section{Algorithm}

The reader should refer to FUN3D documentation ${ }^{5}$ for a description of the baseline algorithm. The presentation that follows highlights algorithm modifications implemented for the hypersonic (high energy - HEFSS) path through the software.

Flux Reconstruction: Reconstruction refers to the algorithm used to derive the right and left states across a cell wall required in the flux definition in finite-volume, approximate Riemann solvers. Limiters refer to the algorithm used to constrain the reconstructed right and left states to prevent introduction of new extrema in the flowfield. Engagement of a limiter typically reduces the local order of accuracy of the reconstructed flux from $2^{\text {nd }}$ to $1^{\text {st }}$ order. Aftosmis et al. discuss the effects of various linear reconstruction techniques on unstructured meshes in Ref. 19. They note an increased degradation of solution quality associated with engagement of limiters on triangular (unstructured) meshes as compared to quadrilateral (structured) meshes. They also note that the use of directional limiting (local engagement of the limiter for each edge direction) as opposed to a global limiting (engagement of the limiter considering all directions emanating from a node) produced higher quality solutions. These observations are consistent with behavior observed in the HEFSS benchmark simulations, to be discussed subsequently.

The basic algorithm uses the divergence theorem (Green-Gauss) for viscous gradients and Roe's method ${ }^{20}$ for inviscid flux:

$$
\mathrm{f}=\frac{1}{2}\left[\mathrm{f}_{\mathrm{L}}+\mathrm{f}_{\mathrm{R}}-\mathcal{R}^{-1}|\Lambda|\left(\mathrm{d} \widetilde{\mathrm{q}}-\mathrm{d} \widetilde{\mathrm{q}}_{\text {lim }}\right)\right]
$$

where

$$
\mathrm{d} \widetilde{\mathrm{q}}=\mathscr{R}\left(\mathrm{q}_{\mathrm{L}}-\mathrm{q}_{\mathrm{R}}\right)
$$

$\mathcal{R}$ and $\mathscr{R}^{-1}$ are the row and column eigenvector matrices of the flux Jacobian and $\Lambda$ is the corresponding diagonal matrix of eigenvalues. The formulation of the Jacobian of $\mathrm{f}$ with respect to $\mathrm{q}$ is presented in Appendix A. Two options are offered here to reconstruct the left (L) and right (R) states. The first (Option 1) uses a least squares gradient $^{21}$ with a limiter function by Barth ${ }^{22}$ or Venkatakrishnan ${ }^{23}$. The term $\mathrm{d}_{\mathrm{q}}$ lim in 9 is identically zero for Option 1. Option 1 is contained in the original FUN3D formulation. The second (Option 2) uses the nodal values for the left and right states. Option 2 achieves second-order spatial accuracy with the Symmetric Total Variation Diminishing $^{24}$ (STVD) scheme applied as a directional limiter (function of edge orientation) as follows: 


$$
\begin{aligned}
& \mathrm{d} \widetilde{\mathrm{q}}_{\lim }=2 \phi\left(\mathrm{p}_{\mathrm{L}}, \mathrm{p}_{\mathrm{R}}\right) \operatorname{minmod}\left(\mathrm{d} \widetilde{\mathrm{q}}_{\mathrm{L}}, \mathrm{d} \widetilde{\mathrm{q}}_{\mathrm{R}}, \mathrm{d} \widetilde{\mathrm{q}}, \frac{1}{4}\left(\mathrm{~d} \widetilde{\mathrm{q}}_{\mathrm{L}}+\mathrm{d} \widetilde{\mathrm{q}}_{\mathrm{R}}\right)\right) \\
& \mathrm{d} \widetilde{\mathrm{q}}_{\mathrm{M}}=\mathscr{R}\left[\mathrm{d} \overrightarrow{\mathrm{s}} \cdot \vec{\nabla} \mathrm{q}_{\mathrm{M}}\right] \\
& \mathrm{d} \overrightarrow{\mathrm{s}}=\left[\left(\mathrm{x}_{\mathrm{L}}-\mathrm{x}_{\mathrm{R}}\right) \overrightarrow{\mathrm{i}}+\left(\mathrm{y}_{\mathrm{L}}-\mathrm{y}_{\mathrm{R}}\right) \overrightarrow{\mathrm{j}}+\left(\mathrm{z}_{\mathrm{L}}-\mathrm{z}_{\mathrm{R}}\right) \overrightarrow{\mathrm{k}}\right]
\end{aligned}
$$

and

$$
\begin{aligned}
& \phi\left(\mathrm{p}_{\mathrm{L}}, \mathrm{p}_{\mathrm{R}}\right)=\left(1-\cos \left(\psi\left(\mathrm{p}_{\text {rat }}\right) \pi\right) / 2\right. \\
& \mathrm{p}_{\text {rat }}=\max \left(\frac{\mathrm{p}_{\mathrm{L}}}{\mathrm{p}_{\mathrm{R}}}, \frac{\mathrm{p}_{\mathrm{R}}}{\mathrm{p}_{\mathrm{L}}}\right)
\end{aligned}
$$

and $\psi$ is 0 if its argument is greater than 4 (fully first order), 1 if its argument is less than 2 (STVD limiting fully engaged), and linearly varying between these limits. The gradients $\vec{\nabla} \mathrm{q}_{\mathrm{M}}$ at nodes $(\mathrm{M}=\mathrm{L}$ or $\mathrm{R})$ are computed using the identical, unlimited least squares formulation of primitive variables from Option 1. The STVD limiting in Option 2 and the Venkatakrishnan limiting in Option 1 are observed to diminish but not eliminate overshoots and undershoots in temperature and pressure in the vicinity of strong shocks. The pressure ratio weighting function, $\phi$, is used to smoothly switch to first-order in the vicinity of strong shocks and under-resolved expansions and to augment the limiting process in Option 2. Options 1 and 2 use identical formulation of the viscous terms.

Eigenvalue Limiting: Elements, $\tilde{\lambda}_{\text {ieqn }}$, of the diagonal eigenvalue matrix $|\Lambda|$ in Eq. 9 are limited from below (entropy fix) ${ }^{25}$ to enhance numerical dissipation across edges where a projected wave speed (Appendix A) approaches zero.

$$
\tilde{\lambda}_{\text {ieqn }}=\left\{\begin{array}{cl}
\left|\lambda_{\text {ieqn }}\right| & \left|\lambda_{\text {ieqn }}\right| \geq 2 \lambda_{\text {ref }} \\
\frac{\lambda_{\text {ieqn }}^{2}}{4 \lambda_{\text {ref }}}+\lambda_{\text {ref }} & \left|\lambda_{\text {ieqn }}\right|<2 \lambda_{\text {ref }}
\end{array}\right\} \quad \text { and } \quad \lambda_{\text {ref }}=\lambda_{0}(\mathrm{a}+|\overrightarrow{\mathrm{u}}|)
$$

Here, $0<\lambda_{0} \leq 1$ for inviscid flows. Application of this limiter across the boundary layer adversely affects computed surface heating and skin friction. ${ }^{26}$ It is easily scaled down in structured grid codes (LAURA) across the boundary layer. A requisite rescaling in the unstructured grid context is based on an evaluation of a local edge Reynolds number where the reference length is a function of radii of inscribed spheres contained in tets surrounding the edge. A loop over cells is executed to compute the inscribed radius for each cell. This value, $r_{\text {ins, vol }}=3$ Volume $/ \sum_{1}^{4}$ Area, is stored at each node of the cell if it is less than current nodal value of $\mathrm{r}_{\text {ins, node }}$. The edge Reynolds number is defined $\operatorname{Re}_{\text {edge }}=\left(\rho \bar{r}_{\text {ins }} / \mu\right)_{\text {edge }}$ where edge values are averaged from the left and right nodal values. The value of $\lambda_{0}$ is reduced by a factor $\min \left[1,\left(\operatorname{Re}_{\text {edge }} / 100\right)^{8}\right]$ in viscous flows. The value of edge Reynolds number across a reasonably resolved boundary layer is expected to vary between 1 and 100; consequently the eigenvalue limiting is effectively turned off in resolved boundary layers and shear layers.

Grid Adaptation: A simple adaptation algorithm has been introduced to reduce cell size and accommodate high aspect ratio tets aligned with captured shocks. The intent is to reduce effects of "stair stepping" as non-aligned, isotropic grids produce "jagged" shocks, leading to erratic entropy fields following the streamlines that cross them. The algorithm is intended to serve as a precursor to adjoint-based adaptation that is available in the baseline FUN3D. A tensile force on each edge is defined $F_{R L}=1+K \frac{\left|g_{R}-g_{L}\right|}{\left(g_{R} g_{L}\right)^{1 / 2}}$. Feature based adaptation is controlled by a user specified function $\mathrm{g}$. The displacement at each node is defined by the sum of the tensile forces along each edge connected to the node. The undamped expression for the displacement of node $\mathrm{L}$ is defined 


$$
x_{j, L}^{n+1}-x_{j, L}^{n}=\delta x_{j, L}=\frac{\sum_{R} F_{R L} x_{j, R}^{n}}{\sum_{R} F_{R L}}-x_{j, L}^{n}
$$

Displacements are damped by a factor 0.5 and limited by the value of $0.5 \mathrm{r}_{\text {ins, node }}$ before updating interior nodes. Updates at boundary surface nodes are constrained to move along the surface. (Grid points are not allowed to move on solid surfaces to prevent corruption of the original CAD surface which is not available as a reference in this simple approach.) The limitation by the inscribed radius is intended to prevent formation of negative volumes in high aspect ratio tets. The adaptation step is applied at a frequency of once per 20 to 50 global relaxation steps.

\section{Results and Discussion}

Inviscid Flow and Entropy Fix: Simulation of an erroneous carbuncle flow from the stagnation region of a blunt body in hypersonic flow with Roe schemes on structured grids can arise from a lack of natural dissipation in extensive regions where the eigenvalues are very small. The underlying scheme looks like a central difference formulation with associated odd-even decoupling. The problem is most evident when structured grids are closely aligned with the captured bow shock. The problem is overcome by limiting the minimum value an eigenvalue in Eq. 9 may assume, as specified in Eq. 12. Originally formulated to suppress rarefaction shocks, the eigenvalue limiting is often called an entropy fix.

Initial tests of hypersonic flow over blunt bodies with unstructured grids did not show evidence of a carbuncle. It was surmised that natural dissipation in the scheme associated with edges that were not generally well aligned with the flow was sufficient to overcome this problem. However, close inspection of the stagnation region in an inviscid, hypersonic flow over an ellipsoid revealed irregularities still existed when Eq. 12 was not used to limit the eigenvalues.

Fig. 1 shows surface pressure distributions in the stagnation region of an ellipsoid for a perfect gas, Mach 6 flow at 30 degrees angle of attack. The simulations are identical except that the simulation displayed on the left employed almost no limiting while the simulation on the right used $\lambda_{0}=1$ in Eq. 12. The limiting acts as a smoother in regions where it is engaged. It responds to the lack of numerical dissipation in the scheme in regions where eigenvalues are small compared to other relevant wave speeds (acoustic, convective). Clearly, some irregularities (non-elliptical shapes) persist in the contours even with the limiter. These are related to uneven shock capturing with a non-aligned grid (discussed in a later section). Though not shown here, solution convergence in leeside flows over bodies at high angle of attack is observed to benefit from the eigenvalue limiting process. In general, eigenvalue limiting is regarded as an essential aspect of the numerical formulation with Roe's method for blunt

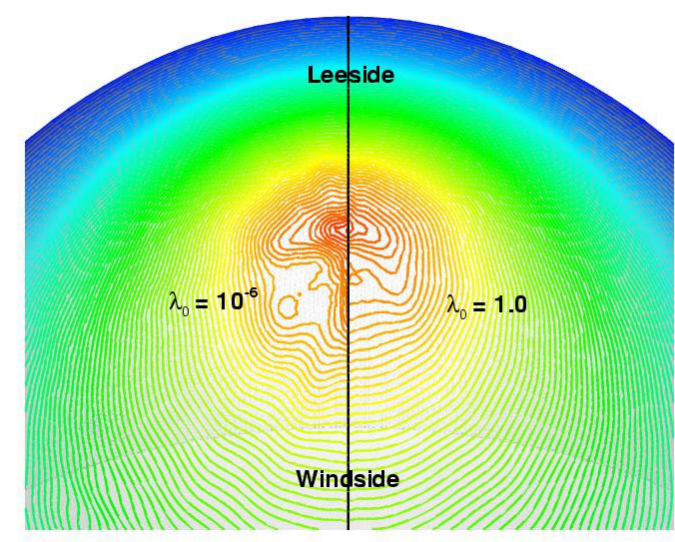

Fig. 1: Effect of eigenvalue limiter on stagnation region pressure contours. over ellipsoid.

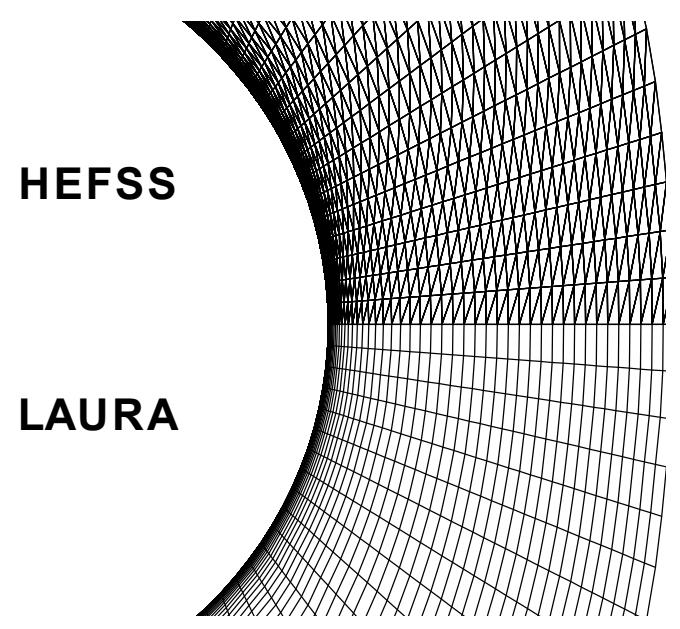

Fig. 2: Structured grid and equivalent uniformly biased unstructured grid in symmetry plane over cylinder for hypersonic test case. 
body, hypersonic flow simulation on unstructured grids, even though the carbuncle phenomenon is not specifically observed. Restriction of the limiters in boundary layers and free shear layers to prevent corruption of computed heating levels is discussed below.

Heating Benchmarks: Benchmark cases for hypersonic flow over a sphere and a cylinder at $\mathrm{V}_{\infty}=5000$ $\mathrm{m} / \mathrm{s}, \rho_{\infty}=0.001 \mathrm{~kg} / \mathrm{m}^{3}, \mathrm{~T}_{\infty}=200 \mathrm{~K}$ (approximately Mach 17) consistent with earlier LAURA benchmarks, have been intensively worked during the HEFSS code development process. The structured LAURA grid, adapted to align with the bow shock, is shown in the bottom part of Fig. 2. The unstructured grid for the HEFSS tests (top of Fig. 2) is formed directly from the structured grid. Both grids are three-dimensional (structured hexahedra (hexes), unstructured tetrahedra (tets)). An unstructured surface grid with ten spanwise cells is shown in Fig. 3. The grid is biased with diagonals running in the same direction. The biasing is intentionally retained to test simulation quality under adverse conditions. Spanwise symmetry of the solution across the bias is easily monitored. Comparisons to the structured grid LAURA results are used as benchmarks in the evaluation process.

The single spanwise cell simulation results with Option 2 (STVD) are shown in Fig. 4. In this case there are no interior nodes in the spanwise direction. The HEFSS results for heating and pressure at surface nodes (indicated by symbol location) on the front and back plane are presented; the pressure symbols on the front and back plane exactly match, the heating levels exhibit a very small offset (less than half a symbol height). Good agreement with the LAURA reference is achieved and the near perfect over plotting of the front and back plane results indicates excellent spanwise constancy. The ten spanwise cell simulation results with Option 2 (STVD) are shown in Figs. 5 and 6. In this case there are nine interior nodes on each spanwise line as shown in Fig. 3. The value at every surface node from the HEFSS simulation is plotted. The eleven spanwise nodes for pressure again show excellent overlap but the heating shows significant spanwise variation approaching the $y=0$ plane (Fig. 5) with the maximum spread in the stagnation region and solution quality improving away from the stagnation region. The average value of both the pressure and heating continue to be in good agreement with the LAURA results. The equivalent result using Option 1 with the Venkatakrishnan limiter is shown in Fig. 7. In this case, pressure constancy is slightly degraded and more

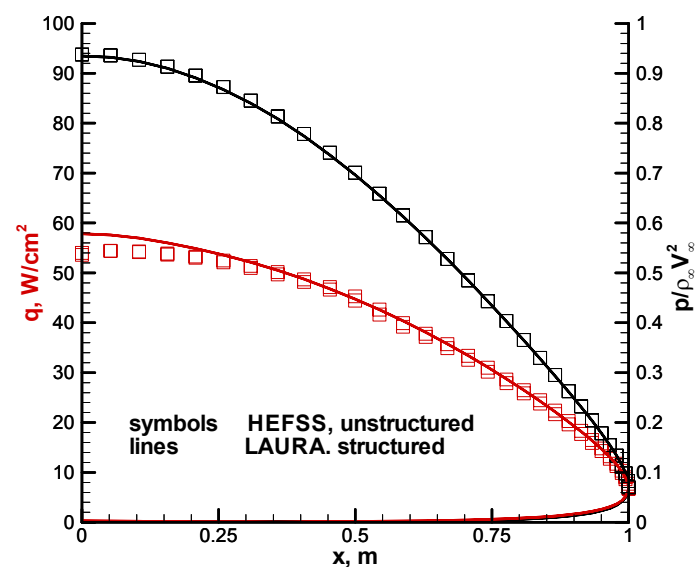

Fig. 4: Pressure and heating distribution for cylinder test case with 3D, uniformly biased, unstructured grid across one spanwise cell.

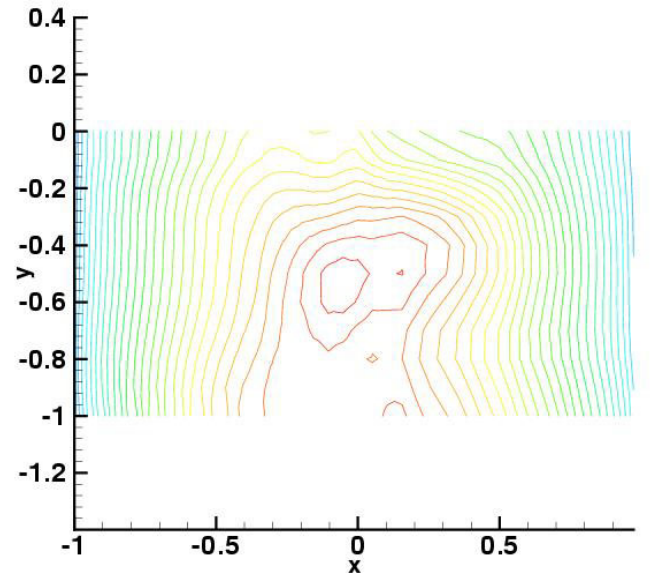

Fig. 5: Heating contours in stagnation region of cylinder with 10 spanwise cells 


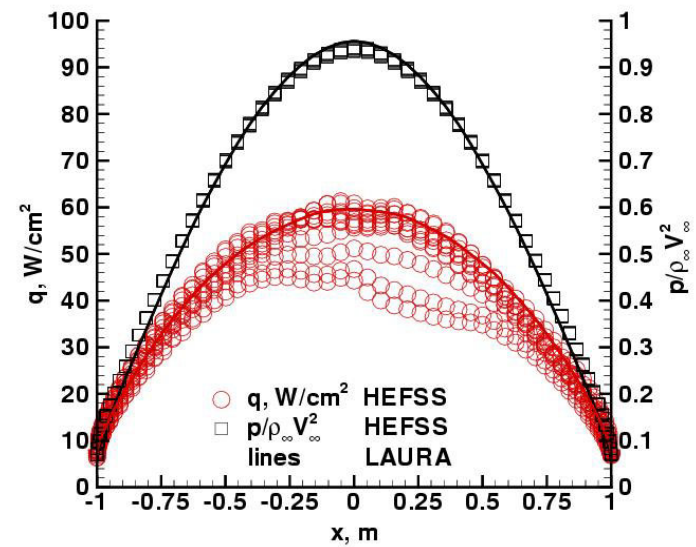

Fig. 6: Pressure and heating distribution for cylinder test case with 3D, uniformly biased, unstructured grid across ten spanwise cells with Option 2.

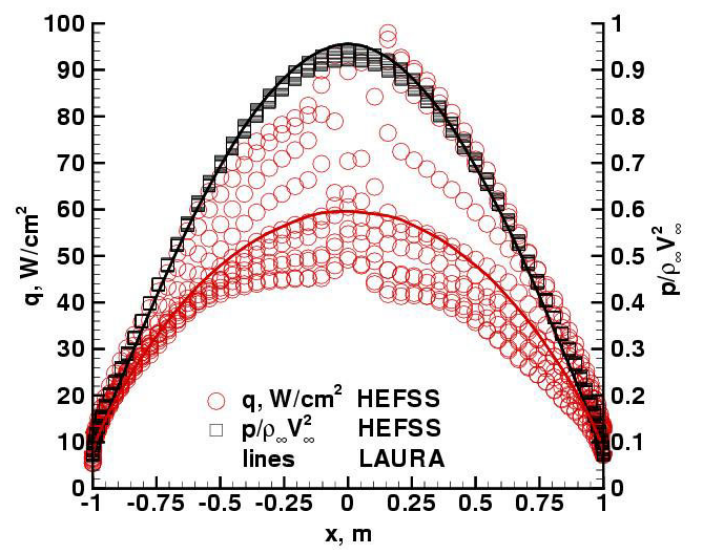

Fig. 7: Pressure and heating distribution for cylinder test case with 3D, uniformly biased, unstructured grid across ten spanwise cells with Option 1.

significant asymmetry in heat transfer is evident. Though not shown, the Option 1 results with the Barth limiter are of poorer quality and appear more like a first-order result. In all cases, the symmetry of shock layer contour plots of pressure, temperature, and Mach number show consistent trends; the spanwise symmetry properties of the Option 2 path are superior to all other tested options.

These results demonstrate that solution quality for stagnation point heating behind strong shocks using tets all the way to the wall is poor using any of the reconstruction options discussed herein. It is unfair to lay all of the blame on the Green-Gauss formulation of viscous gradients across high aspect ratio cells when the results are so obviously sensitive to the higher order inviscid formulation. In any case, the freedom to utilize tets across the entire domain - even to the wall - provides the greatest flexibility for future grid adaptation. Note that the first-order, edge-based reconstruction in Eq. 9 only involves information at nodes R and L. A conjecture is offered that a truly multi-dimensional reconstruction of the flux at every face could overcome some of the simulation quality issues noted above. A multi-dimensional upwind (MDU) algorithm for high speed flows has recently been developed ${ }^{27,28}$; it is not yet known how it would perform on the ten-spanwise-cell cylinder test problem for hypersonic, blunt-body heating.

Reacting Gas Benchmark: The heating benchmark case described above has been repeated for a five species air model in chemical nonequilibrium and thermal equilibrium. Only the single spanwise cell grid is used. A fully catalytic wall boundary condition is approximated by specifying mole fractions at the wall equal to mole fractions in the cold freestream. The equivalent LAURA solution is again utilized as a benchmark. Excellent agreement between LAURA and HEFSS predictions for species mass fraction across the shock layer and boundary layer are observed in Fig. 8. Surface heating, including conduction and diffusion components, show excellent agreement (Fig. 9) outside of the stagnation region. The HEFSS prediction of stagnation point heating is slightly less than LAURA, consistent with results of Fig. 4. Shear stress is

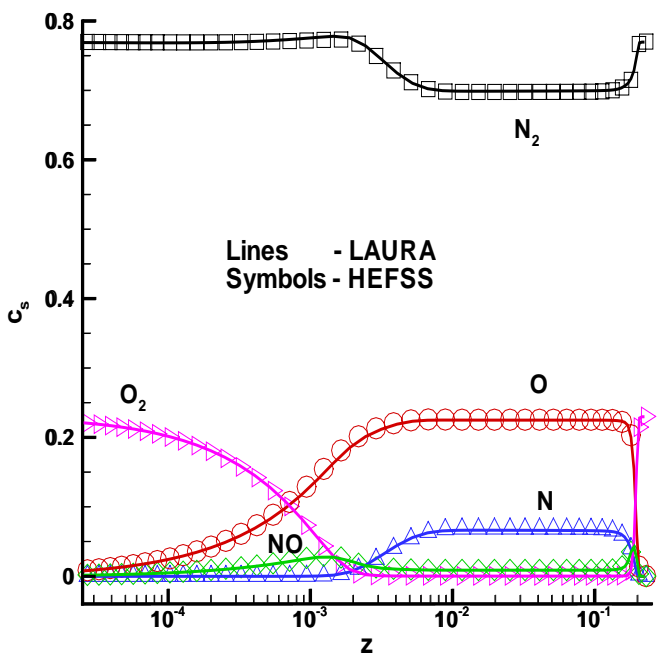

Fig. 8: Species mass fraction distribution across the stagnation streamline. 


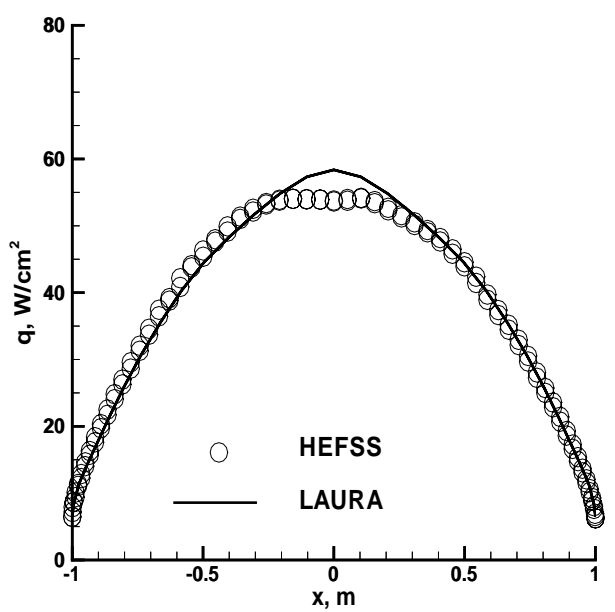

Fig. 9: Heating distribution around cylinder at Mach 17 for reacting air, fully catalytic wall.

grid biasing in that asymmetry is obvious between the left and right side HEFSS results. The peak shear from HEFSS is greater than the LAURA result. Investigation of this issue is ongoing.

Turbulent Flat Plate Benchmark: Turbulent flow of a perfect gas at Mach 6 and Reynolds number 26.38 million over a plat plate is simulated with HEFSS and VULCAN (benchmark). The wall to total temperature ratio is 0.6. Both codes used the same $\mathrm{K}-\omega$ model without compressibility corrections. Transition is initiated at the leading edge of the plate. Comparisons of heating and skin friction coefficient are presented in Fig. 11. The skin friction coefficients are in excellent agreement except at the leading edge where boundary condition implementation differs between a node based (HEFSS) and cell center based (VULCAN) codes. The HEFSS heating is about $10 \%$ lower than VULCAN over most of the plate.

Grid Adaptation Test: The grid adaptation algorithm described in the previous section is tested on the inviscid ellipsoid (Fig. 1). The function $g$ that controls tensile force $F_{R L}$ in Eq.

(13) was keyed to pressure, velocity and temperature. An adaptation frequency of once per 20 relation steps was applied over approximately 5000 steps. A before and after view of the grid on the symmetry plane is shown in Fig. 12. The grid clearly shows the extent of the captured bow shock. The clustering is accommodated with high aspect ratio cells. The corners of the domain are deformed because the algorithm to constrain movement on boundaries smoothes the surface normal at common boundaries. The cleaner shock capture results in significant improvement in the shape of pressure contour lines (Fig. 13) in the stagnation region. (Contrast Fig 13 with Fig. 1.) 


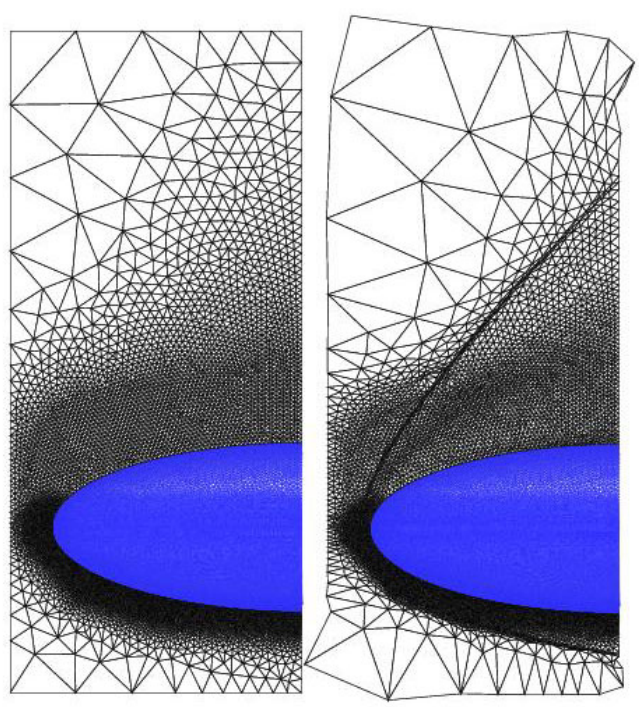

Fig. 12: Original and adapted grids over ellipsoid in symmetry plane.

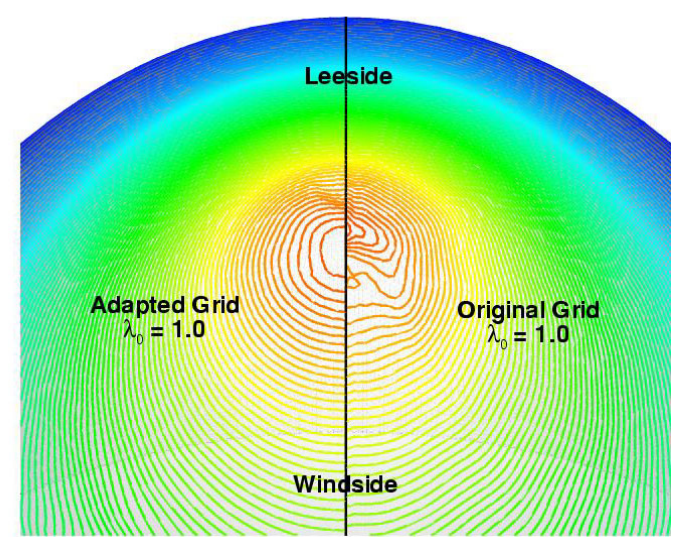

Fig. 13: Pressure contours over ellipsoid with and without grid adaptation.

Shuttle Orbiter: A simulation of hypersonic flow in thermochemical nonequilibrium (5-species air model) over the shuttle orbiter is presented to demonstrate present capabilities using only tets $(1.1$ million nodes) for discretization elements. The original grid was assembled very quickly (1 day) during the Columbia accident investigation using GridEx ${ }^{29}$ software. The simulation was abandoned early on because it became clear that solution quality was not adequate with the existing algorithm. The simulation was revisited for this paper in order to document current capabilities on a reasonably complex problem, especially regarding need for grid adaptation. To repeat a theme developed earlier, we must demonstrate a capability to quickly generate an adequate grid and depend on the software to automatically adapt and refine it. In this case, the viscous near wall grid spacing was based on an existing structured grid solution from LAURA ${ }^{30}$ at Mach 24. (STS-2, $\mathrm{V}_{\infty}=6920 \mathrm{~m} / \mathrm{s}, \rho_{\infty}=5.7510^{-5} \mathrm{~kg} / \mathrm{m}^{3}, \quad \mathrm{~T}_{\infty}=$ $202 \mathrm{~K}, \alpha_{\infty}=39.4 \mathrm{deg}$., and $\left.\mathrm{h}=72.4 \mathrm{~km}\right)$ The grid was marched out from the body in 40 layers. An essentially isotropic grid filled the volume between the viscous, near wall grid and the specified mesh spacing along the outer boundaries (farfield, exit, and symmetry plane).

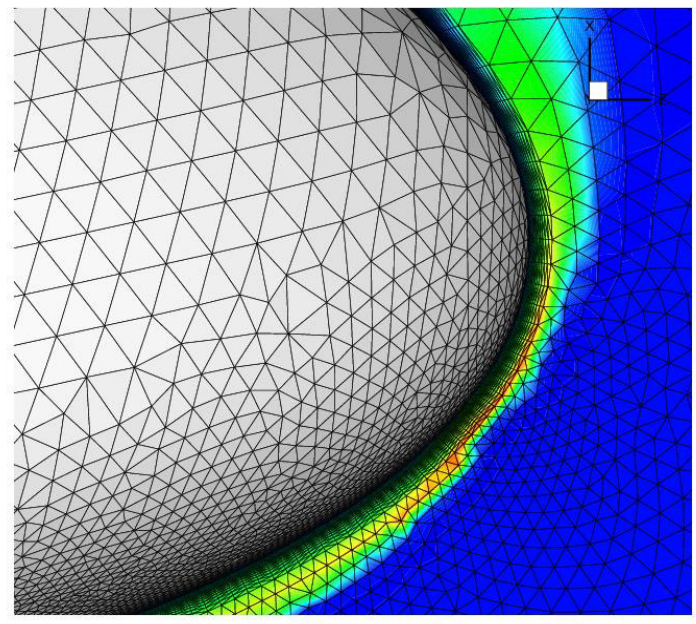

Fig. 14: Temperature contours in plane of symmetry over the nose in the original grid.

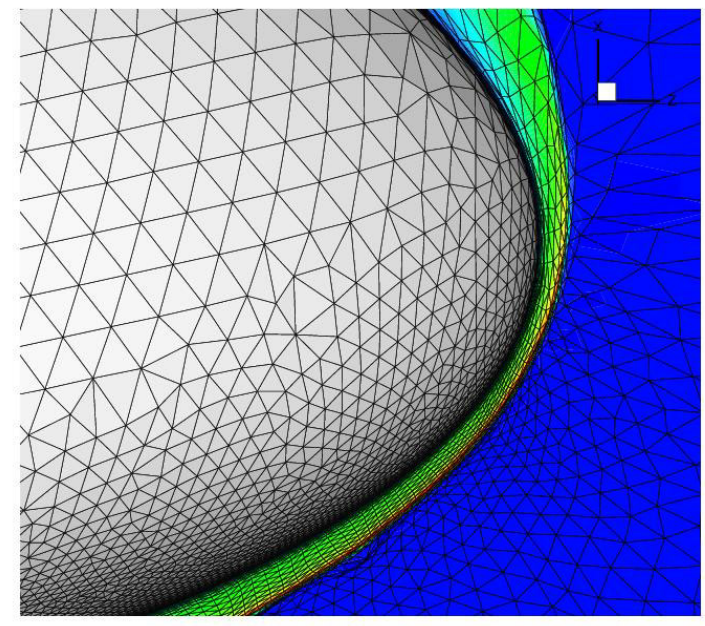

Fig. 15: Temperature contours in plane of symmetry over the nose in the adapted grid. 


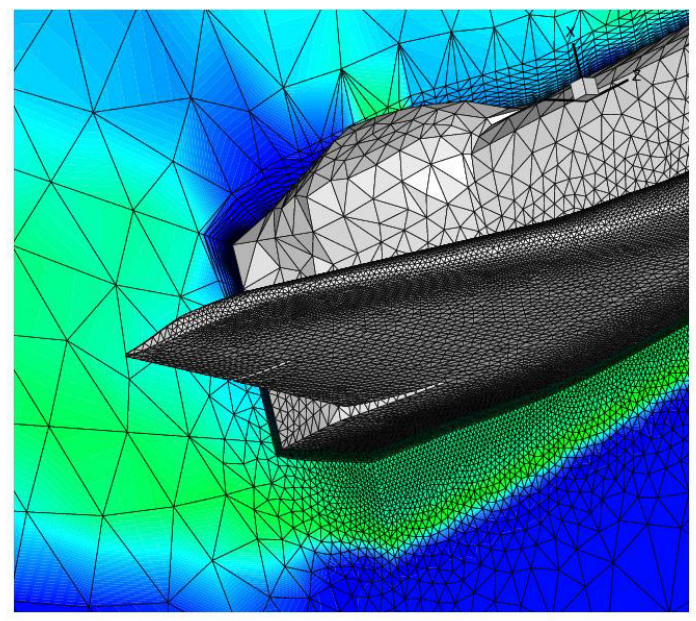

Fig. 16: Temperature contours in plane of symmetry and exit plane behind the trailing edge in the original grid.

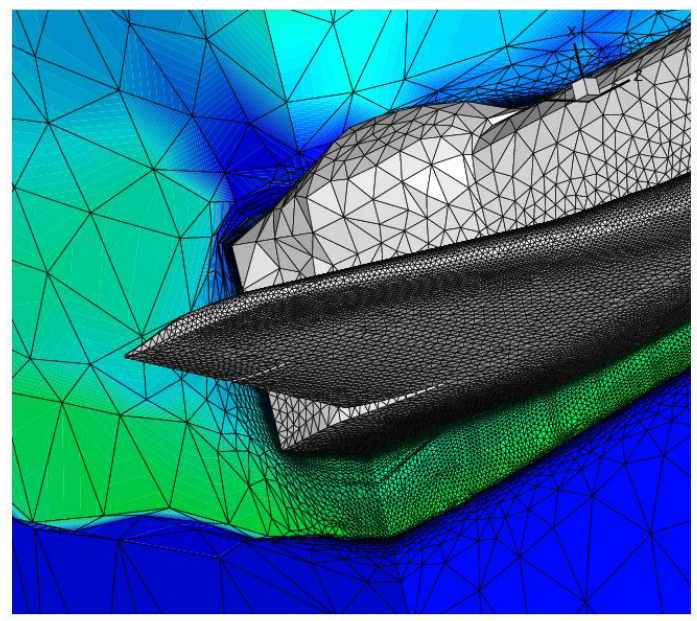

Fig. 17: Temperature contours in plane of symmetry and exit plane behind the trailing edge in the adapted grid.

The grid over the nose in Fig. 14 is clearly inadequate to cleanly capture the bow shock. However, sufficient resolution is provided to enable a sharper capture with high aspect ratio tets in Fig. 15. Further downstream, it is evident that the original grid (Fig. 16) has extremely poor resolution of the wing wake and leeside (no tail in this simulation) over the fuselage. The captured bow shock front is diffuse and wavy. The adapted grid (Fig. 17) again provides a cleaner shock capture and picks up an oblique shock over the deflected body flap (the back end of the shuttle is filled in over the body flap). However, the simple adaptation algorithm is unable to pull in more nodes to resolve vortex structure on the leeside or the wing wake. In fact, the leeside symmetry plane was plagued by an instability that is believed to be caused by very poor resolution of the separated vortex. A tendency for reverse flow at the exit plane between the OMS pods was also encountered; the solution would be reset at locations where this occurred as indicated by a local temperature falling outside a range of $0.1 \mathrm{~K}$ to $30,000 \mathrm{~K}$.

The ability to produce high aspect ratio cells aligned with the captured shock is a great advantage of this simple, spring-based adaptation strategy. However, the strategy as currently implemented cannot perform edge swapping nor can it provide local enrichment of nodes as obviously required on the wake and leeside. Isolated locations were observed where the grid appeared to be drawn to a point in the absence of any apparent driving gradients. This behavior is thought to be a remnant of the grid evolution process and restrictions to grid movement when the inscribed radius of any tet connected to a node gets small. (In the present case, no movement was allowed at any node where the inscribed radius was less than 0.001 inches.) Recent adaptation algorithms developed by Park $^{31}$ will be applied here in an effort to correct these deficiencies.

Windside pressure contours from HEFSS on the adapted grid are compared to the LAURA solution in Fig. 18. Agreement is generally good. The unstructured grid solution included elevon gaps and body flap that were not included in the LAURA solution, thus accounting for differences at the trailing edge. The

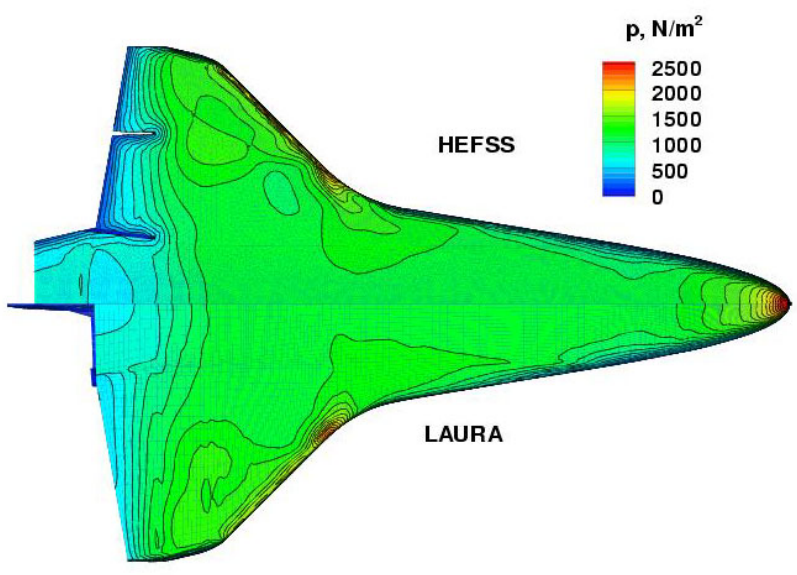

Fig. 18: Windside pressure contours from unstructured HEFSS and structured LAURA. 
nose region is in excellent agreement. The original, pre-adapted grid solution was very erratic looking - much like the behavior shown in Fig. 13 on the ellipsoid.

The leeside pressures in Fig. 19 are in poor agreement, as would be expected given the poor leeside resolution discussed earlier. The compression over the canopy and vortex impingement on the OMS pod is greatly dissipated.

Windside heating distributions are compared in Fig. 20. In this case, contour lines should not be expected to be mirror images because the surface boundary conditions are different. The HEFSS simulation used a constant wall temperature of $500 \mathrm{~K}$ and a fully catalytic wall boundary condition; it does not yet have the option for the finite catalytic, radiative equilibrium boundary condition used in LAURA. These differences do not have a strong influence on windside pressure in Fig. 18. The heating on the HEFSS simulation should be higher than LAURA as generally observed in Fig. 20 with these boundary conditions. However, the HEFSS contour lines are more erratic in nature. Heating near the plane of symmetry shows a wake like behavior downstream of the stagnation region. The wake of the bow shock - wing shock interaction is more sharply defined in the HEFSS solution though contour lines remain somewhat jagged. The high heating around the elevon gaps is exaggerated because of surface grid is too coarse to resolve flow drawn into the gap.

The results of Fig. 20 highlight the challenges remaining to bring HEFSS up to the standards required for vehicle design and analysis. Recall that we intentionally restrict all simulations to use only tets. This restriction arises from a belief that if semistructured grid (hexes and prisms) are required in the near wall layers to achieve good / smooth heating then one is likely masking problems elsewhere in the flowfield

- especially if separated flows or interactions off the surface are present. The assertion here is that if one uses semistructured, high-aspect-ratio elements near the wall then one must also provide an algorithm to automatically generate and align equivalent elements elsewhere in the field as a function of flow physics. Such a grid adaptation algorithm may in fact be the best approach to this problem. The approach pursued here is to develop an algorithm that can provide a quality aerothermal simulation using only tets. The results in this paper highlight our best efforts to that end at this point. Several strategies adopted here (STVD limiting, entropy fix scaled to local metric of cell Reynolds number, high-aspect-ratio grid adaptation) have improved aerothermal simulation for hypersonic flows. The fact that the single spanwise cell cylinder results yield good aerothermal simulations lead us to believe that the problem is not so much that the tets cannot support a good solution but that current algorithms admit discretization errors that manifest themselves more severely in the randomly oriented unstructured grid context than in the 
structured grid context. We speculate that the quasi-one-dimensional, edge oriented, reconstruction used here is the predominant cause of the problem and future work will be directed at this concern.

\section{Concluding Remarks}

The synthesis of physical models for gas chemistry and turbulence from the structured grid codes LAURA and VULCAN into the unstructured grid code FUN3D is described. This suite of modules for hypersonic application is called the High Energy Flow Solver Synthesis (HEFSS). A Symmetric, Total Variation Diminishing (STVD) algorithm and entropy fix (eigenvalue limiter) keyed to local cell Reynolds number are introduced to improve solution quality for hypersonic applications focused on aeroheating. A simple grid-adaptation procedure is incorporated within the flow solver to reduce effects of jagged captured shocks on shock layer profiles. Simulations of flow over an ellipsoid (perfect gas, inviscid), Shuttle Orbiter (viscous, chemical nonequilibrium) and comparisons to the structured grid solvers LAURA (cylinder, Shuttle Orbiter) and VULCAN (turbulent flow on a flat plate) are presented to show current capabilities.

Early in the testing phase of HEFSS it became apparent that stagnation region heating was poorly predicted. The poor predictions are much more a result of inviscid algorithm deficiencies than shortcomings associated with exclusive use of tetrahedral elements across the shock and boundary layer. Two sets of numerical results support this assertion. First, single-spanwise-cell cylinder tests on a strongly biased, unstructured grid for both single-species and five-species flow in thermochemical nonequilibrium compared well with heating benchmarks from a structured grid code. In contrast, the ten-spanwise-cell cylinder tests (same grid as the single-spanwise-cell case repeated across the span) showed a marked degradation in heating contours in the stagnation region, apparently arising from spanwise flow admitted with the additional degrees of freedom. If grid were the primary culprit, one would not expect a good solution in the single-spanwise-cell tests. Second, numerical tests on the inviscid flux formulation (eigenvalue limiting, reconstruction options) had significant effects on the stagnation region heating with little effect outside the stagnation region. These results indicate that a primary cause of heating degradation arises from how the inviscid flow gets processed crossing the shock and the shock layer to the boundary-layer edge; and not from poor formulation of viscous terms across the boundary layer itself. Indeed, inviscid flow tests on an ellipsoid showed pressure contour irregularities (occurring only in the stagnation region) were fixed by application of an entropy fix and better grid alignment. If pressure (and entropy) at the boundary layer edge is not correct, there is no chance to get good prediction of heating.

A Symmetric Total Variation Diminishing (STVD) algorithm with cell Reynolds number scaled eigenvalue limiting produced the best quality heating in the stagnation region of several approaches tested. However, all inviscid formulations tested utilized a quasi-one-dimensional reconstruction and none of them produced exceptionally good predictions of heating in the stagnation region. The STVD approach as applied herein will admit new maxima and minima across a captured shock and requires application of an auxiliary pressure switch for stability. Research continues on defining an inviscid reconstruction algorithm that will yield consistently good hypersonic stagnation heating using only tetrahedral elements across the entire shock layer.

\section{Acknowledgements}

Michael Park and Bill Kleb of NASA Langley Research Center developed the grid used for the Shuttle Orbiter.

\section{Appendix A: Characteristic Variables and Jacobians}

Formation of characteristic variables and inviscid flux Jacobians closely follows that in LAURA ${ }^{11}$. However, the reconstruction variable set uses primitive variables rather than conservative variables. The Jacobian of the inviscid flux vector with respect to conserved variables is expressed below in Eq. A1 as a function of its eigenvalues. Note that subscripts $s$ and $r$ refer to species equations, subscripts $i$ and $\mathrm{j}$ refer to momentum equations, and subscripts $v$ and $v$ refer to supplemental energy mode equations. A single, total energy equation is also included. The two equation turbulence models are not explicitly included here. Their form looks very much like the species or energy mode rows. Additional terms arise in the momentum and energy equations if turbulent pressure in the Reynolds stress tensor is treated as part of the inviscid flux contribution to pressure (rather than a source term which is not involved in the upwind discretization). 
$\mathrm{r}$ columns $\quad \mathrm{j}$ columns $\quad 1$ column $\quad \mathrm{v}$ column

$$
\begin{aligned}
& \text { s rows } \tilde{\lambda}_{1} \delta_{\mathrm{sr}}-\mathrm{c}_{\mathrm{s}}\left(\tilde{\lambda}_{2} \mathrm{U}-\tilde{\lambda}_{0} \tilde{\gamma}_{\mathrm{r}}\right) \quad \tilde{\lambda}_{0} \beta \mathrm{u}_{\mathrm{j}} \mathrm{c}_{\mathrm{s}}+\tilde{\lambda}_{2} \mathrm{c}_{\mathrm{s}} \mathrm{n}_{\mathrm{j}} \quad-\tilde{\lambda}_{0} \beta \mathrm{c}_{\mathrm{s}} \quad-\tilde{\lambda}_{0} \varphi_{\mathrm{v}} \mathrm{c}_{\mathrm{s}}
\end{aligned}
$$

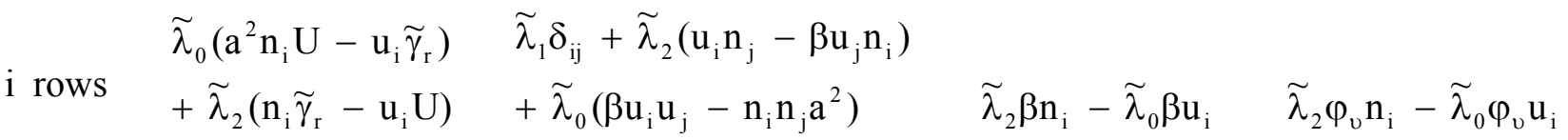

$$
\begin{aligned}
& 1 \text { row } \quad \tilde{\lambda}_{0}\left(\mathrm{U}^{2} \mathrm{a}^{2}-\mathrm{H} \widetilde{\gamma}_{\mathrm{r}}\right) \quad \tilde{\lambda}_{2}\left(\mathrm{Hn}_{\mathrm{j}}-\beta \mathrm{u}_{\mathrm{j}} \mathrm{U}\right) \\
& +\tilde{\lambda}_{2}\left(\mathrm{U} \widetilde{\gamma}_{\mathrm{r}}-\mathrm{HU}\right)+\tilde{\lambda}_{0}\left(\beta \mathrm{u}_{\mathrm{j}} \mathrm{H}-\mathrm{Ua}^{2} \mathrm{n}_{\mathrm{j}}\right) \quad \tilde{\lambda}_{1}+\tilde{\lambda}_{2} \beta \mathrm{U}-\tilde{\lambda}_{0} \beta \mathrm{H} \quad \tilde{\lambda}_{2} \mathrm{U} \varphi_{\mathrm{v}}-\tilde{\lambda}_{0} \mathrm{H} \varphi_{\mathrm{v}} \\
& \mathrm{v} \text { rows }-\tilde{\lambda}_{2} \mathrm{e}_{\mathrm{v}} \mathrm{U}-\tilde{\lambda}_{0} \mathrm{e}_{\mathrm{v}} \quad \tilde{\lambda}_{0} \beta \mathrm{u}_{\mathrm{j}} \mathrm{e}_{\mathrm{v}}+\tilde{\lambda}_{2} \mathrm{e}_{\mathrm{v}} \mathrm{n}_{\mathrm{j}} \quad-\tilde{\lambda}_{0} \beta \mathrm{e}_{\mathrm{v}} \quad \tilde{\lambda}_{1} \delta_{\mathrm{v}}-\tilde{\lambda}_{0} \varphi_{\mathrm{v}} \mathrm{e}_{\mathrm{v}}
\end{aligned}
$$

The eigenvalues of the Jacobian A are: $\lambda_{1}=U=u_{i} n_{i}$ and $\lambda_{2,3}=U \pm a$. The functions of eigenvalues for the Jacobians $\mathrm{A}$ and $|\mathrm{A}|$ are now defined:

$$
\begin{aligned}
& \tilde{\lambda}_{1}=\left\{\begin{array}{lll}
\lambda_{1} & \text { for } & \mathrm{A} \\
\left|\lambda_{1}\right| & & |\mathrm{A}|
\end{array}\right.
\end{aligned}
$$

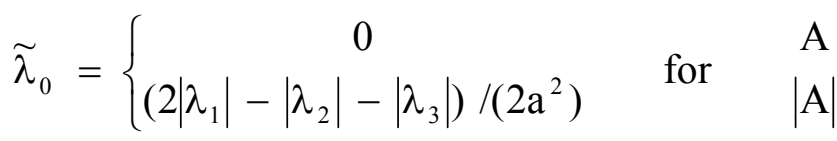

$$
\begin{aligned}
& \tilde{\lambda}_{2}=\left\{\begin{array}{c}
1 \\
\left(\left|\lambda_{2}\right|-\left|\lambda_{3}\right|\right) /(2 \mathrm{a})
\end{array} \quad \text { for } \quad \begin{array}{c}
\mathrm{A} \\
|\mathrm{A}|
\end{array}\right.
\end{aligned}
$$

The elements of $\mathrm{d} \widetilde{\mathrm{q}}$ in Eq. 10 are defined:

$$
\begin{aligned}
& d \widetilde{q}_{s}=a^{2} d \rho_{s}+c_{s}\left(-\widetilde{\gamma}_{r} d \rho_{r}+\beta u_{j} d \rho u_{j}-\beta d \rho E-\varphi_{v} d \rho e_{v}\right)=a^{2} d \rho_{s}-c_{s} d p \\
& \mathrm{~d} \widetilde{\mathrm{q}}_{\mathrm{s}+1}=-\mathrm{Vd} \rho+\ell_{\mathrm{j}} \mathrm{d} \rho \mathrm{u}_{\mathrm{j}}=\rho \ell_{\mathrm{j}} \mathrm{du} \mathrm{u}_{\mathrm{j}}=\rho \mathrm{dV} \\
& \mathrm{d} \widetilde{\mathrm{q}}_{\mathrm{s}+2}=-\mathrm{Wd} \rho+\mathrm{m}_{\mathrm{j}} \mathrm{d} \rho \mathrm{u}_{\mathrm{j}}=\rho \mathrm{m}_{\mathrm{j}} \mathrm{du} \mathrm{u}_{\mathrm{j}}=\rho \mathrm{dW} \\
& \mathrm{d} \widetilde{\mathrm{q}}_{\mathrm{s}+3}=\left(\widetilde{\gamma}_{\mathrm{r}} \mathrm{d} \rho_{\mathrm{r}}-\beta \mathrm{u}_{\mathrm{j}} \mathrm{d} \rho \mathrm{u}_{\mathrm{j}}+\beta \rho \mathrm{E}+\varphi_{\mathrm{v}} \mathrm{d} \rho \mathrm{e}_{\mathrm{v}}\right)+\mathrm{a}\left(-\mathrm{Ud} \rho+\mathrm{n}_{\mathrm{j}} \mathrm{d} \rho \mathrm{u}_{\mathrm{j}}\right)=\mathrm{dp}+\rho \mathrm{adU} \\
& \mathrm{d} \widetilde{\mathrm{q}}_{\mathrm{s}+4}=\left(\widetilde{\gamma}_{\mathrm{r}} \mathrm{d} \rho_{\mathrm{r}}-\beta \mathrm{u}_{\mathrm{j}} \mathrm{d} \rho \mathrm{u}_{\mathrm{j}}+\beta \rho \mathrm{E}+\varphi_{\mathrm{v}} \mathrm{d} \rho \mathrm{e}_{\mathrm{v}}\right)-\mathrm{a}\left(-\mathrm{Ud} \rho+\mathrm{n}_{\mathrm{j}} \mathrm{d} \rho \mathrm{u}_{\mathrm{j}}\right)=\mathrm{dp}-\rho \mathrm{ddU} \\
& \mathrm{d} \widetilde{\mathrm{q}}_{\mathrm{s}+4+\mathrm{v}}=\mathrm{e}_{\mathrm{v}}\left(-\widetilde{\gamma}_{\mathrm{r}} \mathrm{d} \rho_{\mathrm{r}}+\beta \mathrm{u}_{\mathrm{j}} \mathrm{d} \rho \mathrm{u}_{\mathrm{j}}-\beta \mathrm{d} \rho \mathrm{E}-\varphi_{\mathrm{v}} \mathrm{d} \rho \mathrm{e}_{\mathrm{v}}\right)+\mathrm{a}^{2} \mathrm{~d} \rho \mathrm{e}_{\mathrm{v}}=\mathrm{a}^{2} \mathrm{~d} \rho \mathrm{e}_{\mathrm{v}}-\mathrm{e}_{\mathrm{v}} \mathrm{dp}
\end{aligned}
$$

where we have used $d p=\beta\left(d \rho E-u_{j} d \rho u_{j}\right)+\varphi_{v} d \rho e_{v}+\widetilde{\gamma}_{r} d \rho_{r} \quad$ (again neglecting optional inclusion of turbulent pressure term $\beta_{\mathrm{t}} \rho \mathrm{K}$ as part of the upwind discretization).

\section{References}

${ }^{1}$ Gnoffo, P. A.; Weilmuenster, K. J.; Hamilton, H. H. II; Olynick, D. R.; Venkatapathy, E.: “Computational Aerothermodynamic Design Issues for Hypersonic Vehicles," JSR, Vol. 36, No. 1, pp 21-43, Jan. 1999.

${ }^{2}$ Gnoffo, Peter A.: "Planetary-Entry Gas Dynamics," Ann. Rev. Fluid Mech. 1999, 31:459-94.

${ }^{3}$ Cheatwood, F. M. and Gnoffo, P. A.: "User's Manual for the Langley Aerothermodynamic Upwind Relaxation Algorithm (LAURA)", NASA TM 4674, April 1996.

${ }^{4}$ White, J. A.; Morrison, J. H.: “A Pseudo-Temporal Multi-Grid Relaxation Scheme for Solving the Parabolized Navier-Stokes Equations,” AIAA Paper 99-3360, June 1999. 
${ }^{5}$ Anderson, W. K.; Bonhaus, D. L.: “An Implicit Upwind Algorithm for Computing Turbulent Flows on

Unstructured Grids," Computers and Fluids, Vol. 23, No. 1, pp 1-21, 1994.

${ }^{6}$ Thomas, J. L. et al.: "Opportunities for Breakthroughs in Large-Scale Computational Simulation and Design," NASA TM 2002-211747, June 2002.

${ }^{7}$ Gnoffo, P. A.: "Computational Fluid Dynamics Technology for Hypersonic Applications," AIAA-3259, July 2003.

${ }^{8}$ McBride, B. J. and Gordon, S.: "Computer Program for Calculation of Complex Chemical Equilibrium

Compositions and Applications. II. Users Manual and Program Description," NASA RP 1311, June 1996.

${ }^{9}$ Gordon, S. and McBride, B. J.: "Thermodynamic Data to $20000 \mathrm{~K}$ for Monatomic Gases," NASA TP-1999-

208523, June 1999.

${ }^{10}$ Park, C.: "Assessment of Two-Temperature Kinetic Model for Ionizing Air. AIAA Paper 87-1574, June 1987.

${ }^{11}$ Gnoffo, P. A.; Gupta, R. N.; Shinn, J. L.: "Conservation Equations and Physical Models for Hypersonic Air Flows in Thermal and Chemical Nonequilibrium," NASA Technical Paper 2867, Feb. 1989.

${ }^{12}$ Park, C.: Review of Chemical-Kinetic Problems of Future NASA Missions, I: Earth Entries” JTHT, Vol. 7, No. 3 pp385-398, 1993.

${ }^{13}$ Park, C; Howe, J. T.; Jaffe, R. L.; Candler, G. V.: "Review of Chemical-Kinetic Problems of Future NASA missions, II: Mars Entries," JTHT, Vol. 8, No. 1, pp 9-23, 1994.

${ }^{14}$ Gupta, R. N.; Lee, K. P.; Thompson, R. A.: "Calculations and Curve Fits of Thermodynamic and Transport Properties for Equilibrium Air to 30000K,” NASA RP 1260, 1991.

${ }^{15}$ Gupta, R. N.; Yos, J. M.; Thompson, R. A.; Lee, K. P.: "A Review of Reaction Rates and Thermodynamic and Transport Properties for an 11-Species Air Model for Chemical and Thermal Nonequilibrium Calculations to 30,000K," NASA RP 1232.

${ }^{16}$ Sutton, K.; Gnoffo, P. A.: "Multicomponent Diffusion with Application to Computational Aerothermodynamics," AIAA Paper 98-2575.

${ }^{17}$ Rumsey, C.L. and Gatski, T.B., "Recent Turbulence Model Advances Applied to Multielement Airfoil Computations," AIAA Paper 2000-4323, August 2000.

${ }^{18}$ Wilcox, D. C., Turbulence Modeling for CFD, 2nd Ed., DCW Industries, La Canada, 1998.

${ }^{19}$ Aftosmis, M.; Gaitonde, D.; Tavares, T. S.: "Behavior of Linear Reconstruction Techniques on Unstructured Meshes," AIAA J., Vol. 33, No. 11, Nov. 1995, pp2038-2049.

${ }^{20}$ Roe, P. L.: “Approximate Riemann Solvers, Parameter Vectors, and Difference Schemes,” J. Comput. Phys., vol. 43, no. 2, Oct. 1981, pp. 357-372.

${ }^{21}$ Barth, T. J.: "Numerical Aspects of Computing High-Reynolds Number Flows on Unstructured Meshes," AIAA Paper 91-0721, 1991.

${ }^{22}$ Barth, T. J.; Jespersen, D. C.: "The Design and Application of Upwind Schemes on Unstructured Meshes," AIAA Paper 89-0366, Jan. 1989.

${ }^{23}$ Venkatakrishnan, V.: "Convergence to Steady-State Solutions of the Euler Equations on Unstructured Grids with Limiters," J. Comp. Phys, 118:120-130, 1995.

${ }^{24}$ Yee, H. C.: "Construction of Explicit and Implicit Symmetric TVD Schemes and Their Applications," J. Comput. Phys., vol. 68, no. 1, Jan. 1987, pp. 151-179

${ }^{25}$ Harten, Ami: "High Resolution Schemes for Hyperbolic Conservation Laws," J. Comput. Phys., vol 49, no. 2, Feb. 1983, pp. 357-393.

${ }^{26}$ Gnoffo, Peter A.: "An Upwind-Biased, Point-Implicit Relaxation Algorithm for Viscous, Compressible PerfectGas Flows," NASA TP 2953, Feb. 1990.

${ }^{27}$ Van der Weide, E. "Compressible Flow Simulation On Unstructured Grids Using Multi-Dimensional Upwind Schemes," Ph.D thesis, Aero. Eng., Delft University of Technology, 1998.

${ }^{28}$ Houtman, E.M.; Van der Weide E.; Deconinck, H. and Bakker, P.O.: "Computational Analysis of Base Flow/Jet Plume Interaction," Proc. 3d European Symp. on Aerothermodynamics for Space Vehicles, ESA SP-426, pp 605$612,1998$.

${ }^{29}$ Jones, W. T.: “An Open Framework for Unstructured Grid Generation,” AIAA Paper 2002-3192, June 2002.

${ }^{30}$ Gnoffo, P. A.; Weilmuenster, K. J.; Alter, S. J.: "Multiblock Analysis for Shuttle Orbiter Re-entry Heating from Mach 24 to Mach 12," JSR, Vol. 31, No. 3, pp 367-377, May-June 1994.

${ }^{31}$ Park, M. A.: "Three-Dimensional Turbulent RANS Adjoint-Based Error Correction," AIAA 2003-3849, June 2003. 